\title{
¿Es competitiva la pequeña y mediana industria de la Región Zuliana?
}

\author{
Jenny Romero de Cuba* \\ Ana M. Acosta \\ Elizabeth Bohorquez
}

\section{Resumen}

El actual proceso de globalización ha servido para revitalizar el papel que juegan las pequeñas y medianas industrias en otros países. Las nuevas exigencias del mercado hacen que la PYMI pueda atenderlo de manera más oportuna y con las especificaciones requeridas, pues su rápida capacidad de adaptación a los cambios del entorno y la flexibilidad de su estructura organizativa, le confiere una importante ventaja competitiva. Tomando en cuenta este contexto, el presente artículo se plantea como objetivo determinar el grado de adecuación de la PYMI del Estado Zulia (Venezuela) al proceso de globalización. La metodología utilizada se fundamenta en la revisión de estadísticas regionales correspondientes al período 1989 - 1994, y en el cálculo de índices que permiten medir la eficiencia y productividad de estas unidades. El análisis de los resultados revela inquietantes indicios en cuanto a su capacidad competitiva. En general, se trata de un aparato productivo concentrado en la industria tradicional, cuya productividad es poco significativa. Aunado a ésto se encuentra un proceso continuo de desinversión que compromete seriamente su capacidad futura de producción, y las aleja cada vez más de una inserción exitosa. Para poder revertir esta situación se requiere el desarrollo de planes y programas

Fecibido: 27-2-98 . Aceptado: 26-3-98

* Magister en Gerencia de Empresas. Investigadora del Instituto de Investigaciones de la Facultad de Ciencias Económicas y Sociales (FCES) de la Universidad del Zulia (LUZ). Autor para la correspondencia. Telf, 061-596513.

** Magister en Políticas Públicas. Investigadora del instituto de Investigaciones de la Facultad de Ciencias Económicas y Sociales de la Universidad del Zulia.

*** Economista. Asistente de Investigación del Instituto de Investigaciones de la Facultad de Ciencias Económicas y Sociales de la Universidad del Zulia, tesista de la Maestría en Gerencia de Empresas. 
estratégicos dirigidos a mejorar los niveles de productividad y calidad del sector, cuya responsabilidad debe ser compartida por el sector público y privado.

Palabras clave: Pequeña y mediana industria, globalización, productividad, competitividad.

\title{
Are Small and Medium Sized Industries Competetive in the Zulia Region?
}

\begin{abstract}
The present process of globalization has served to revitalize the role which is played by small and medium sized industries (SMSI) in other countries. The new market requirements permit the SMSI to react more opportunely and with the specifications required since their rapid capability of adjustment to environmental changes and the flexibility of their organizational structures give them an important competitive edge. On the basis of this situation, this paper proposes the objective of determining the grade of adjustment to globalization demonstrated by the the Venezuelan Zulia State SMSI. The methodology used was based on the revision of regional statistics corresponding to the years 1989-1994, and on the calculation of indexes which permited the measurement of industrial efficiency and productivity of the Zulian SMSI. The analysis of the results reveals a disquieting indication of their competitive capacity. In general, we are faced with a traditional industrial productive apparatus, whose productivity is of little significance. We also find a continuous process of non or disinvestment which seriously compromises future productive capacity, and effectively dampens the possibility of successful global insertion. To reverse the process, the development of strategic plans and programs directed towards bettering productivity and quality levels is necessary. The responsability for this should be shared by the public and private sector.
\end{abstract}

Key words: Small and Medium Sized Industry, Globalization, Productivity, Competetivity 


\section{Introducción}

Las dos últimas décadas han caracterizado notables cambios en la economía mundial, cuya tendencia actual se manifiesta en el proceso de globalización impulsado por el desarrollo tecnológico y comunicacional, el cual ha permitido una mayor movilización de los recursos productivos, bienes y servicios de un pais a otro.

Esta nueva realidad ha modificado radicalmente el tipo de relación comercial sostenida por la mayoría de las naciones, hecho que a su vez las ha obligado a insertarse en los procesos de integración económica. En otras palabras, se ha avanzado hacia un modelo de integración más abierto, en el cual la competencia tiene lugar entre países de diferentes grados de desarrollo, y donde la participación exitosa exige como requisito esencial la obtención de estándares elevados de desempeño industrial.

Frente a este escenario los paises de menor desarrollo industrial, entre ellos Venezuela, confrontan un gran desafio que les impone reordenar su estructura productiva de acuerdo con los patrones de competitividad establecidos en el mercado mundial.

Dentro de este contexto, la pequeña y mediana industria (PYMI) está llamada a jugar un papel protagónico en la conformación de una nueva estructura industrial, tal como ha sucedido en economías altamente competitivas como la japonesa, la italiana y la coreana, donde ha quedado demostrado que la flexibilidad organizativa y la rápida adaptabilidad a los cambios del entorno, le confieren a la PYMI una importante ventaja en el co- mercio internacional. Por esta razon, se ha venido observando una tendencia mundial hacia el empequeñecimiento de las grandes empresas, ésto con la intención de concentrarse en segmentos de mercado en los que se tenga mayor fortaleza.

A pesar de lo anteriormente señalado y de la importancia estratégica que como negocio han adquirido estas empresas, este estudio permite acotar que en la región zuliana la competitividad de la pequeña y mediana industria es cuestionable, debido fundamentalmente al poco valor que añaden a la producción industrial del estado, lo cual está íntimamente relacionado con la baja productividad de este sector y el continuo proceso de descapitalización al que se ha visto sometido durante los últimos años; aunado a ello se encuentra la ausencia de vocación exportadora y el desconocimiento de las oportunidades que se están generando dentro del contexto de la globalización.

\section{La pequeña y mediana industria y su inserción en la economia global}

El fenómeno de la globalización enfrenta al mundo a una nueva era comercial y de segmentación de los mercados, que sin duda alguna, constituye una gran ventaja para la PYMI, debido a su capacidad de adaptación a los cambios del entorno y al estrecho contacto con sus clientes, lo cual les permite atenderlos en forma más oportuna y con las especificaciones requeridas.

Asimismo, es necesario mencionar el auge de las nuevas tecnologías de fa- 
bricación que hacen posible que no sólo a través de las grandes corporaciones se pueda ser competitivo. La automatización flexible a diferencia de la automatización rígida, posibilita la superación del viejo paradigma de economías de escala. "Ya no es necesaria la producción de grandes volúmenes para minimizar los costos unitarios, ahora es factible la producción rentable de pequeños lotes" ( $\mathrm{Pi}$ zani, 1993;16).

Lo anteriormente expuesto refleja los argumentos que actualmente existen en torno al importante papel que pueden jugar las pequeñas y medianas industrias en la economía moderna. Son numerosos los ejemplos, y cada día crecen aún más las experiencias que así lo confirman. En este sentido, se hace necesaria la revisión de algunos procesos aleccionadores sobre el comportamiento de este sector, de manera que puedan servir de base para explicar por qué la PYMI de ciertos países ha podido incursionar con éxito en el mercado mundial.

Experiencia japonesa: Japón es considerada una de las economías más exitosas en el comercio mundial; parte de este logro se debe a la aplicación del enfoque de la programación industrial, en el cual el sector empresarial se encuentra perfectamente articulado a un Estado que proyecta una visión de largo plazo.

Dentro de este engranaje la pequeña y mediana empresa (PYME) cumple un rol muy importante, pues representa aproximadamente el $99 \%$ de los establecimientos industriales y comerciales, además de absorber el $80 \%$ de la fuerza laboral del país en 1991 (Hosono, 1992;2).
Para poder alcanzar el éxito la PYMl japonesa se ha afianzado en una serie de políticas públicas que han sido aplicadas tomando en cuenta el momento y situación por los que atraviesa la economía, pudiendo distinguirse tres grandes fases que caracterizan el apoyo estatal:

La primera fase, que se inicia con la finalización de la segunda guerra mundial hasta finales de los años cincuenta, se caracterizó por proteger a las pequeñas industrias de las grandes fábricas. La segunda, que se extiende hasta los inicios de los años setenta se orientó hacia el fomento y estímulo de la PYMI a través de la asistencia financiera, trato preferencial en materia de impuestos y subsidios para el desarrollo de la investigación y la tecnología; y la tercera fase comprende desde los años setenta hasta el presente, y se caracteriza por la utilización de la informática y la elaboración de productos de alta calidad (Rosales, 1995; 9).

También es conveniente considerar que una de las políticas que mayor impacto ha tenido en el fortalecimiento de estas empresas fue la política del Benchmarking. Esta política consistió en fomentar entre los directivos de las empresas la actividad de mirar fuera de sus organizaciones y tratar de adaptar los procesos de las empresas competidoras más eficientes para fortalecer la propia. Otro factor que contribuyó al logro de este objetivo es el sistema adoptado por las grandes empresas japonesas de subcontratación de empresas pequeñas y medianas como proveedoras de un gran número de partes y piezas a la industria manufacturera, siendo el caso más conocido 
el sector de la industria automotriz (Yokokura, 1984;18).

Finalmente, hay que considerar los rasgos culturales propios de la sociedad japonesa, donde existe una cultura de grupo, hábitos de trabajo, disciplina y visión de futuro.

Experiencia Italiana: Italia ha desarrollado su PYMl en forma más espontánea y menos dirigida, caracterizada por una menor participación del Estado, pero en donde se advierten equilibrios sociales y la economía se halla inserta en el mercado europeo.

La PYME contribuye con aproximadamente el $70 \%$ del producto interno bruto para 1991, existiendo una alta participación de esta en el sector manufacturero, con un total de 700.000 empresas que realizan casi el $70 \%$ de la producción de bienes, y aproximadamente el $70 \%$ de las ventas en el exterior (Bassetti, 1994; 2).

Entre las razones del éxito internacional de la PYMI Italiana Bassetty (1994; 3-7) destaca:

- La relativa facilidad de acceso al mercado internacional, a raíz de la creación de un área de intercambio libre en Europa desde el decenio de 1950 y de la liberalización del intercambio mundial con el GATT.

- Existencia por un largo período de tiempo de numerosos sistemas de protección de los mercados locales, hecho que permitió crecer y prosperar a la PYMI.

- Flexibilidad en el uso de los recursos y la consiguiente rapidez para adaptarse a una demanda de mercado internacional variable y sujeta a mercados cada vez más segmentados y con sectores muy especificos.

- Un nivel de cultura técnica que hizo posible la entrada en la actividad manufacturera moderna, permitiéndoles a los pequeños industriales realizar continuas innovaciones (basadas en el conocimiento profundo de los materiales, de las técnicas de proceso, etc.) que los mantuvieron siempre a un nivel competitivo.

- Fuerza de trabajo calificada, formada en escuelas técnicas financiadas con la colaboración de los organismos públicos y empresariales.

- Atención dedicada a las nuevas tecnologías de proceso y la capacidad de hallar nuevas aplicaciones para las mismas.

- Presencia de una tupida red de ciudades pequeñas y medianas en casi todo el territorio, ciudades ricas de tradiciones y de capacidades artesanales, manufactureras y comerciales.

- Existencia en todo el territorio de un gran número de bancos de dimensiones pequeñas, que han suministrado flujos de crédito para sostener la pequeña empresa.

La experiencia italiana es útil por ser un sistema abierto, dotado de una organización flexible, y en donde los "jointventure" internacionales o alianzas estratégicas forman la base de su éxito. 


\section{Experiencia Coreana: En Corea} al igual que en Japón las políticas estatales dirigidas a la PYMI han pasado por varias etapas (Baek, 1993; 561). La primera se inicia en los años sesenta, y se orientó fundamentaimente a incentivar la creación de empresas pequeñas con el objeto de detener la caída del empleo; para ese entonces, la política industrial centraba su atención en la producción y exportación de productos estratégicos como los de las industrias pesada y química, dominada por industrias de gran escala. La segunda etapa abarca los años setenta e inicios de los ochenta, que se caracterizó por el predominio de políticas dirigidas a alcanzar la estabilidad operativa de la PYMI y la búsqueda de nuevos mercados.

Los resultados de éstas políticas se evidenciaron en el corto plazo, registrándose un crecimiento del número de empresas al igual que en otros indicadores como el valor agregado. La tercera etapa que corresponde a los años ochenta, en donde la política industrial del gobierno coreano da un giro significativo, pasando del apoyo selectivo a uno más amplio y funcional para mejorar el mecanismo de asignación de recursos destinados a incrementar las inversiones en investigación y desarrollo.

En la actualidad los esfuerzos están orientados hacia la modernización de las empresas a través del uso intensivo de tecnologías de avanzada como la de nuevos materiales, robótica y biotecnología; ésto con el objetivo último de transformar la estructura industrial concentrada en la utilización de mano de obra en forma intensiva; este modelo las hizo competitivas a nivel mundial en los renglones de textiles, calzados, juguetes, etc. Sin embargo, en los últimos años sus exportaciones se han visto reducidas como consecuencia de la pérdida de competitividad experimentada a nivel mundial por estas industrias.

\section{Experiencia Latinoamericana:} En América Latina la pequeña y mediana industria presenta una situación muy heterogénea, donde se distinguen economías con diferentes grados de desarrollo industrial. A pesar de las diferencias estructurales existentes en los procesos de crecimiento y acumulación de estos países, la PYMI enfrenta en términos generales una situación de subsistencia, originada en gran parte por su baja productividad y débil competitividad. No obstante, en contadas ocasiones se incorpora a la competencia moderna en condiciones favorables.

En México, por ejemplo, la política de apertura comercial tuvo una incidencia negativa en el sector de la PYMI, debido a que la competencia se dio en forma desigual, siendo afectadas principalmente por los países de Asía, los cuales inundaron el mercado mexicano con productos de alta calidad a precios notablemente inferiores. A pesar de esta situación, se produjo un hecho favorable como fue la concertación lograda entre empresarios y gobierno en relación a una reestructuración estratégica de la capacidad productiva del sector (Sánchez, 1993; 539).

En el caso de Chile las reestructuraciones económicas llevadas a cabo por el gobierno (acelerada apertura comercial, liberalización financiera, reducción del sector público, entre otras) han preci- 
pitado a un número significativo de empresas a la quiebra, pero tras la recuperación, las empresas sobrevivientes se han propuesto llevar a cabo un proceso de modernización y de integración para lograr el anhelado desarrollo del sector. En los últimos años estos esfuerzos se han visto cristalizados, dado que se han incrementado notablemente las exportaciones y se han ampliado los mercados de exportación. Prueba de ello, es que en el periodo 1973 - 1991 el número de productos vendidos en el exterior subió de 208 a 3000 , los mercados de destino de 60 a 149 y la cantidad de empresas exportadoras de 210 a 5200 , correspondiendo al sector de la PYMI unas 4000 empresas (Henriquez, 1993; 547), Sin embargo, es conveniente acotar que el crecimiento del sector externo descansa más sobre el dinamismo de otros sectores, como el agrícola, que del propio sector industrial.

La experiencia de Brasil tiene en común con la de otras economías latinoamericanas, la existencia de una PYMI con un nivel tecnológico limitado, que utiliza para su financiamiento preferentemente capital propio. No obstante, en los últimos años la PYMI brasileña ha concentrado sus esfuerzos en mejorar el nivel tecnológico, al mismo tiempo que ha incrementado sus demandas de apoyo estatal en las áreas de financiamiento, asistencia técnica y fomento a las exportaciones. Cabe destacar que estas políticas han dado los resultados esperados; como muestra se tiene el incremento observado en sus exportaciones, sobre todo, en productos que concentran un alto valor agregado como la fabricación de equipos de transporte, en los cuales la
PYMI participa mediante el sistema de subcontratación.

Con relación a Venezuela, se puede afirmar que ésta al igual que el resto de América Latina, ha tenido que adaptarse violentamente a los procesos de integración económica y a la tendencia mundial de la economía de mercado.

Como se recordará, durante más de treinta años los gobiernos venezolanos impuisaron abiertamente una política de fomento a las actividades industriales basada en la sustitución de importaciones. Una de las premisas fundamentales de dicho proceso fue la política proteccionista, cuyo uso indiscriminado configuró un aparato industrial ineficiente y poco competitivo, tanto a nivel nacional como internacional.

Es a finales de la década de los sesenta cuando aparecen los primeros sintomas de agotamiento de la expansión industrial. Las excesivas importaciones de bienes de capital y tecnología, y la gran cantidad de recursos destinados a la política proteccionista, terminaron por causar graves problemas en las finanzas públicas. Por otra parte, la estrechez del mercado interno se constituyó en un freno estructural al proceso de acumulación, y acentuó con el tiempo el crecimiento de la capacidad ociosa, el desperdicio de capital y el consumo suntuario. Paradójicamente, coexistió una situación de baja productividad en la industria, al mismo tiempo que se registraron tasas de ganancias extraordinariamente altas, escenario que fue sostenido gracias a los abundantes recursos provenientes del petróleo. Es solamente a partir de 1978 cuando el proceso presenta importantes fluctuaciones, configurándose una fase 
recesiva que se prolonga hasta nuestros días.

El deterioro de los precios del petróleo y la puesta en marcha de la política de apentura económica causaron un doble impacto en la mayoria de las empresas venezolanas; por un lado, el gobierno ya no dispone de los recursos financieros para seguir con el sistema de subsidios y exoneraciones. Por el otro, una gran parte de las empresas no estaban preparadas para asumir los cambios, produciéndose el cierre de un elevado número de ellas; incluso para muchas el objetivo principal ha sido y sigue siendo la supervivencia. No obstante, cabe resaltar que algunas empresas reaccionaron favorablemente a los cambios, abriéndose un abanico de oportunidades en el mercado internacional.

En el estrato de la pequeña y mediana industria, las consecuencias son mucho más severas y evidencian una baja sustancial en la participación de la producción total del sector industrial, una agudización de los problemas estructurales, tales como el aumento de la desarticulación de la estructura industrial, escaso desarrollo tecnológico, deficiencia en la capacidad gerencial, técnica y organizativa, entre otros.

\section{Replanteo de la competitividad}

Los procesos económicos y políticos en marcha han llevado a replantear el concepto de la competitividad. En el pasado este término estuvo íntimamente asociado al concepto de ventajas comparativas, en donde se destacaba que la su- premacía de una nación se fundamentaba en la abundancia de sus recursos naturales.

Hoy en día el concepto de competitividad ha adquirido diversas interpretaciones. Para algunos la competitividad de una empresa puede ser definida como "la capacidad que tiene ésta de obtener una posición ventajosa en el mercado interno, ofrecer productos de calidad e incursionar exitosamente en los mercados globalizados" (Alvarez, 1994;151).

Para otros, como es el caso de la Organización para la Cooperación y Desarrollo Económico (OCDE), la definición gira en torno al dinamismo y a la disposición a competir de las empresas, así como su capacidad para invertir e innovar. En términos generales el análisis de la competitividad toma en cuenta una serie de indicadores que de alguna u otra forma miden la productividad industrial. Para alcanzar este objetivo a nivel de la pequeña y mediana industria de la región zuliana se estudio el comportamiento de algunas variables claves como son: el producto o valor agregado, el valor de las inversiones y la eficiencia de dicho sector, especiaimente en términos de productividad.

\section{El aporte de la PYMY Zuliana}

Los datos aportados por la Encuesta Industrial para la región zuliana en el período 1989 - 1994 revelan un balance negativo en el desempeño productivo de la pequeña y mediana industria. En términos generales, se observa un comportamiento irregular del producto, el cual se caracteriza por presentar una leve caída en los primeros años, seguida de una no- 
table recuperación, que finaliza en 1994 con una drástica caída de más del $20 \%$. Esta tendencia tan fluctuante en el valor agregado de la PYMI constituye uno de los efectos más dramáticos producidos por las políticas de ajustes, lo cual aunado a la inestabilidad política y económica imperante durante el periodo, se convirtieron en factores críticos para los pequeños y medianos industriales (ver figura № 1).

Al analizar la participación de la PYMl en el producto manufacturero zuliano se evidencia que la baja contribución de ésta, ejerce un impacto negativo en la región al tiempo que pone al descubierto el rezago que la misma presenta con res- pecto a la gran industria. Lo anterior se corrobora al observar que menos del $20 \%$ del producto industrial zuliano es aportado por la PYMI en 1989, situación que se agrava en 1991 cuando alcanza el valor de $10,15 \%$. Lo verdaderamente resaltante de éstas cifras es que la PYMl concentra alrededor del $90 \%$ de los establecimientos industriales pero su contribución al producto es insignificante (ver tabla № 1). Este comportamiento demuestra el incipiente desarrollo logrado por este sector, situación que contrasta con el extraordinario desarrollo mostrado por la PYMl en países como Japón, donde ésta aporta alrededor del $50 \%$ del producto industrial (Hosono, 1992;3).

\section{Figura № 1}

Producto Industrial por Tipos de Industria según Estratos de Ocupación

Tasas de Crecimiento

Zulia 1989 - 1994

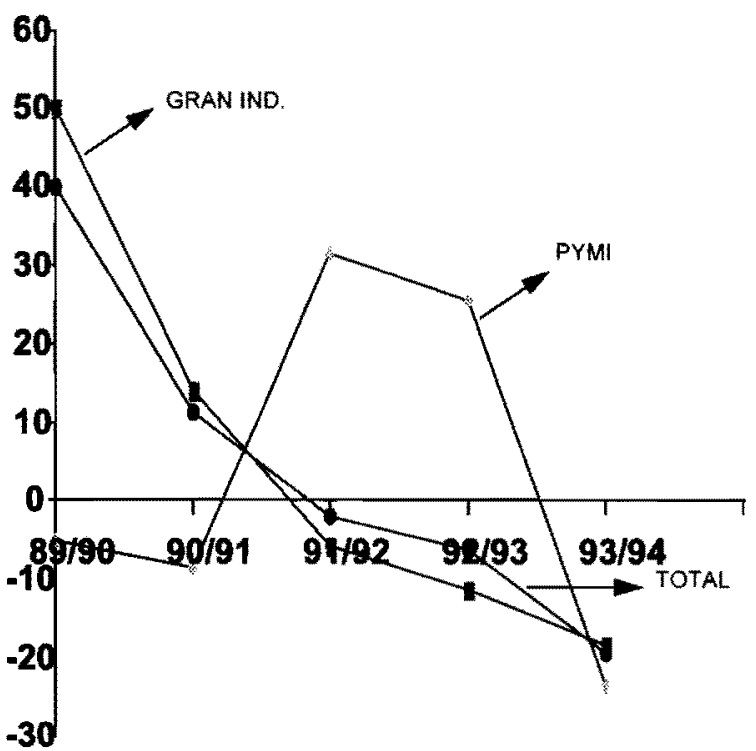

Fuente: Cálculos Propios en Base a la Informacion de la Encuesta Industrial. Años 1989 - 1994. 
Tabla $N^{2} 1$

Región Zuliana: Producto Industrial

Por Estratos de Ocupación

Cifras Relativas

Período 1989 - 1994

\begin{tabular}{ccccccc}
\hline Años & Total & Gran & Med.Sup. & Med.Int. & Pequeña & PYMI \\
\hline 1989 & 100,00 & 81,74 & 9,51 & 4,78 & 3,97 & 18,26 \\
1990 & 100,00 & 87,63 & 5,39 & 4,02 & 2,96 & 12,37 \\
1991 & 100,00 & 89,85 & 4,45 & 3,05 & 2,65 & 10,15 \\
1992 & 100,00 & 86,38 & 7,06 & 3,84 & 2,72 & 13,62 \\
1993 & 100,00 & 81,76 & 10,12 & 5,20 & 2,92 & 18,24 \\
1994 & 100,00 & 82,69 & 7,67 & 4,92 & 4,72 & 17,31 \\
\hline
\end{tabular}

Fuente: O.C.E.I. Encuesta Industrial. Años 1989 - 1994.

Con relación a la distribución del producto industrial por tipo de industrias, se observa que es la industria tradicional la que mayor valor aporta a la PYMI zuliana, pues en el año 1989 genera el $36,42 \%$ del producto de dichas empresas, y para 1994 se acerca al $50 \%$. Siendo la agrupación de alimentos, bebidas y tabaco la más dinámica, dado que concentra aproximadamente el $80 \%$ de la producción de la industria tradicional. En el estrato de la gran industria el sector tradicional aporta algo más de la tercera parte, con tendencia a perder importancia. (verfiguras $\mathrm{N}^{\text {os. }}$. 2 y 3 ).

El elevado porcentaje de la industria tradicional constituye un rasgo estructural de la PYMI venezolana, la cual se concentra en actividades que requieren un menor nivel tecnológico; hecho que justifica en parte su baja contribución al valor agregado y configura en el período analizado un retroceso en su estructura productiva, situación que las aleja cada vez más de una inserción exitosa en el comercio mundial, dada la pérdida de competitividad experimentada por estas industrias en los últimos años.

La industria mecánica constituye la segunda fuente generadora de producto para la PYMI, pues aporta en el año 1989 más de la tercera parte del valor agregado total de la misma. Se debe reconocer que este porcentaje de participación es bastante considerable si se toma en cuenta que la contribución de la gran industria es menor al $8 \%$ en esta actividad. Esta importante participación se explica por la notable influencia que ejerce la industria petrolera como demandante de piezas y partes metálicas fabricadas por esta industria, caracterizada por el predominio de pequeños y medianos establecimientos.

Sin embargo, para el año 1994 se observa una notable disminución de un tercio en el producto de esta industria; es necesario advertir los efectos negativos 
Figura $N^{\circ} 2$

Estructura Porcentual del Producto

De la PYMI por tipos de Industrias

Zulia 1989 Y 1994

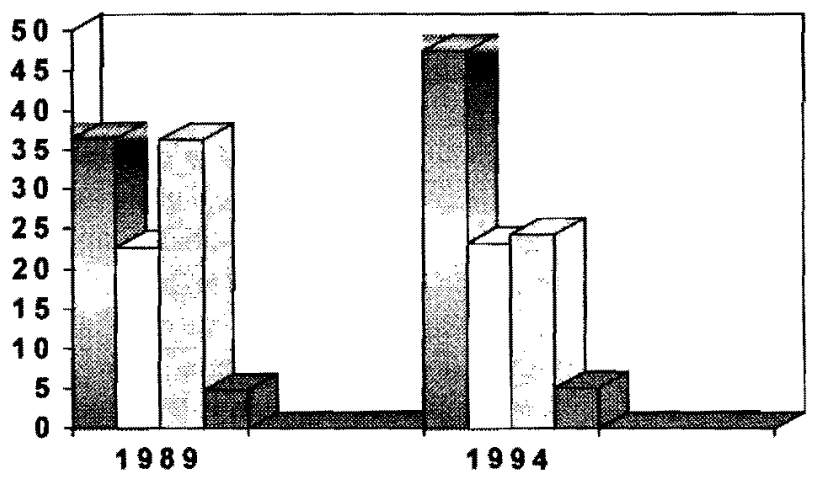

DTRADICIONAL 口INTERM EDIA MECANICA RESIDUAL

Fuente: Calculos propios en base a la informacion de la encuesta Industrial. Años 1989 - 1994.

Figura $\mathrm{N}^{\circ} 3$

Estructura Porcentual del Producto

De la Gran Industria por Tipos de Industrias

Zulia 1989 Y 1994

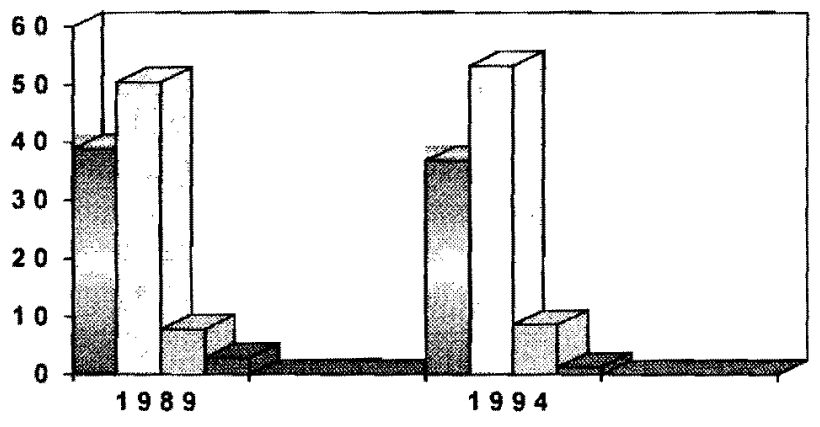

WTRADICIONAL
$\square I N T E R M E D I A$
WECANICA
TRESIDUAL

Fuente: calculos propios en base a la informacion de la encuesta industrial. AÑOS 1989 - 1994. 
que ello genera en la economía zuliana, dado que estas industrias merecen un tratamiento especial, ya que son de alguna forma, un indicador de la capacidad de autoabastecimiento en materia de bienes de capital. Asimismo, el porcentaje de participación de esta industria en Venezuela es considerado insuficiente si se compara con economías altamente tecnificadas como la alemana, japonesa y norteamericana, donde el sector metalmecánico aporta alrededor del $40 \%$ del producto manufacturero total. En estas naciones el sector de los bienes de capital juega un papel fundamental en la competitividad, que le ha permitido mantener una sólida posición de liderazgo en el comercio mundial (Garcia, 1994;8).

La contribución de la industria intermedia en el producto total generado por la PYMI se mantiene por encima del $20 \%$, presentando en 1994 una leve mejoria. La gran industria también presenta un comportamiento favorable en el sector de productos intermedios, el más importante - superior al $50 \%$ - durante el período estudiado, situación que obedece al impulso observado en las ramas de las industrias metálicas básicas, abastecedoras por excelencia de insumos al sector petrolero.

\section{Descapitalización de la PYMI en el Zulia}

Otra de las variables que presenta un comportamiento negativo es la inversión. Esta afirmación tiene como fundamento la significativa reducción registrada en los niveles de inversión de la PYMI zuliana, cuya caída se ubica en el período de estudio cerca al $14 \%$. Este proceso de descapitalización presentado por la PYMI en los últimos años amplía cada vez más la brecha que la separa de los países de mayor desarrollo industrial; en consecuencia el esfuerzo requerido para lograr su despegue industrial debe ser mayor, debido a la elevada tasa de obsolescencia presentada por este sector (Cámara de Industriales del estado Zulia, 1993;16). Entre las causas que explican la situación presentada a nivel de esta variable se encuentran las dificultades existentes en la obtención de financiamiento.

Según el VI Plan de Desarrollo Regional, uno de los problemas más graves a los cuales se enfrenta la PYMI se relaciona con las políticas credicticias de la banca comercial, la cual concede préstamos a corto plazo (un máximo de cuatro años y con tasa de interés del mercado). Estos últimos, se caracterizaron durante los años analizados por ser en extremos elevados, lo que hizo prácticamente prohibitivo el acceso al crédito por parte de este sector. Otras dificultades que también han frenado la inversión están relacionadas con la insuficiencia de recursos otorgados a los organismos gubernamentales financiadores de la PYMI en la región, como la Corporación para el Desarrollo de la Pequeña y la Mediana Industria (CORPOINDUSTRIA), Corporación para el Desarrollo del Estado Zulia (CORPOZULIA) y Fondo de Crédito Industrial (FONCREI), los cuales no disponen de suficientes recursos monetarios como para que se pueda desarrollar este importante sector (Morales, 1997; 90). De igual manera, se evidencian fallas en los criterios manejados por dichos organismos para el otorgamiento de créditos, en vista de que los mismos son aprobados 
sin tomar en cuenta las necesidades y potencialidades de la región zuliana, es decir, no se toma en cuenta la heterogeneidad estructural inherente a estas unidades, enmarcándolas dentro de un mismo esquema de financiamiento sin considerar las diferencias existentes entre las distintas ramas y actividades (Romero, 1994;15).

Este problema exige que los escasos recursos públicos sean administrados de la manera más eficaz, por lo cual se deberia optar por el establecimiento de prioridades basadas en el estudio del dinamismo y potencialidades de cada sector; sólo asi se podrá alcanzar un mayor desarrollo industrial en la región.

El panorama anteriormente descrito compromete seriamente la capacidad futura de producción del sector y amenaza en un corto plazo con el cierre y quiebra de numerosos establecimientos. En tal sentido es necesario advertir que el problema no es sólo de sobrevivir dentro de este escenario, sino de crecer y desarrollarse como empresa, para lo cual se requiere la inversión de grandes volúmenes de capital a fin de alcanzar las innovaciones tecnológicas imprescindibles para adaptarse a tiempo al nuevo paradigma de interrelación comercial y de negocios.

Por esta razón, es necesario que se implemente una política industrial en la cual se otorgue un rol principal al desarrollo cientlfico tecnológico, lo cual permitirá la innovación en los procesos, en los productos y en la gestión dentro de las industrias.

Según una investigación realizada por el Consejo de Desarrollo Regional de la Pequeña y Mediana Industria del esta- do Bolívar, en Venezuela la incorporación del sector privado a la investigación y desarrollo, y la gestión oficial en el logro de ese objetivo no han tenido éxito, porque no se ha vinculado la ciencia y la tecnología al sector privado, y éste no ha sido capaz de financiar la investigación y desarrollo de sus empresas.

\section{Perspectivas de la eficiencia industrial}

Uno de los problemas más frecuentes de las pequeñas y medianas industrias está relacionado con la productividad; por tal razón esta sección tiene como finalidad determinar la productividad de dicho sector en la región zuliana, tomando como base diversos criterios que varian según sea el objeto de su medición y al factor productivo al cual se aplica. Para ello se analiza la productividad de la mano de obra y la eficiencia en la utilización de los recursos productivos.

La productividad de la mano de obra es un índice que se obtiene a través de la relación Valor Agregado/Empleo Industrial y muestra la cantidad de producto generado por persona ocupada.

La figura $^{\circ} 4$ permite visualizar el comportamiento que sigue esta relación. Los datos confirman que la productividad de la PYMI fue notablemente inferior en el período de análisis, pues en promedio es cuatro veces menor a la que presenta el estrato de la gran industria. A pesar de la débil productividad que caracteriza al sector, es importante reconocer que este indicador muestra una evolución más favorable en el caso de los pequeñas y medianas industrias. 
Figura N*4

Productividad de la Mano de Obra

Por Estratos de Ocupación

Zulia 1989 - 1994

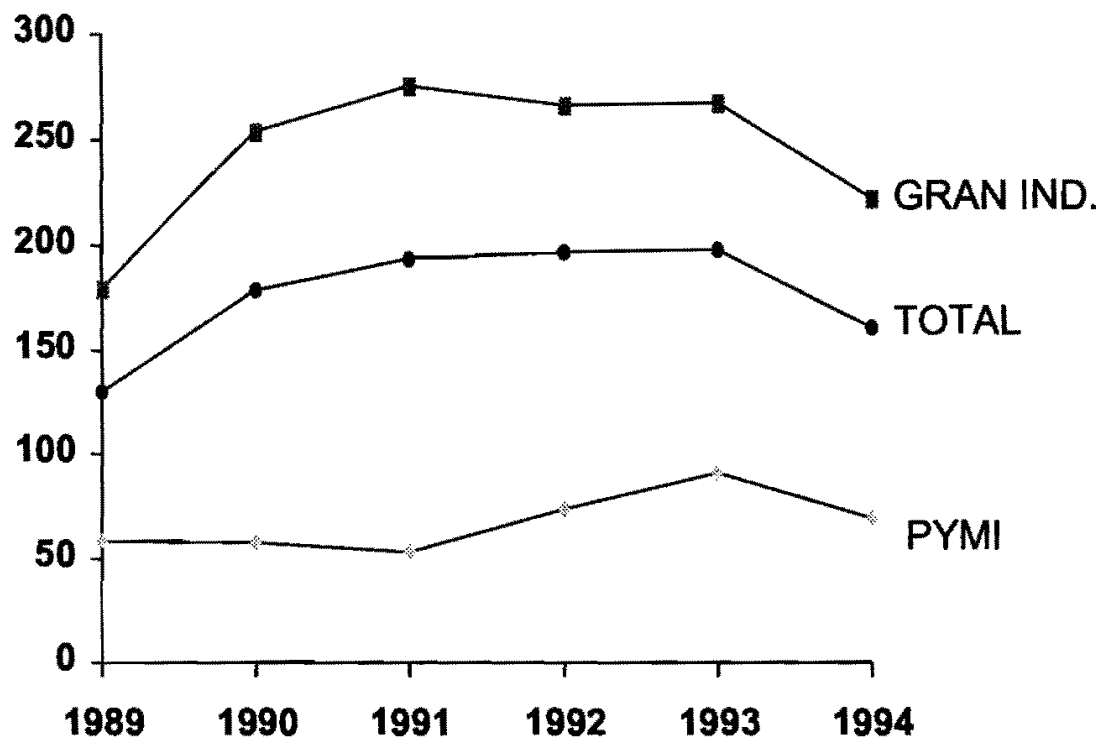

Fuente: Calculos propios en base a la informacion de la encuesta industrial. Años 1989 - 1994.

Cuando se analiza la productividad de la PYMI por agrupación industrial se observa que esta relación es mayor en las industrias mecánicas, siendo la rama de construcción de maquinarias la que presenta un mayor peso (ver tabla $\mathrm{N}^{\circ} 2$ ). Es necesario resaltar que estas industrias a pesar de ser las más afectadas por las nuevas politicas económicas fueron capaces de experimentar una leve mejoría de la productividad del trabajo.
En orden de importancia le sigue la industria intermedia cuya productividad refleja un aumento significativo. En este tipo de industria la rama más dinámica corresponde a los productos químicos y derivados del petróleo y caucho. Cabe subrayar, que estas empresas pese a haber disminuido su parque fabril, y por ende, el empleo generado, logran incrementar sus niveles de productividad, demostrando con ello que son las empresas 
Tabla $N^{2} 2$

Productividad de la Mano de Obra

Por Tipo de Industria y Estratos de Ocupacion

Zulia 1989 - 1994

\begin{tabular}{lrcccccc}
\hline & \multicolumn{3}{c}{1989} & \multicolumn{3}{c}{1994} \\
\hline Actividades & Total & Gran. Ind. & PY MI & Total & Gran Ind. & PYMI \\
Tradicional & 108,78 & 158,35 & 43,57 & 119,90 & 167,86 & 58,18 \\
Intermedias & 201,09 & 252,24 & 66,45 & 303,78 & 380,62 & 94,43 \\
Mécanicas & 85,66 & 83,93 & 87,39 & 102,42 & 109,95 & 91,76 \\
Residual & 83,05 & 154,00 & 37,48 & 66,43 & 101,95 & 47,29 \\
\hline
\end{tabular}

Fuente: Calculos propios en base a la informacion de la encuesta industrial. Años 1989 - 1994.

más competitivas del estrato de la PYMI zuliana (Romero y Acosta, 1997;65)

En to concerniente a la industria tradicional se aprecia que esta relación es una de las más bajas dentro del conjunto, vale decir, que por estar menos tecnificadas emplean en forma intensiva el factor mano de obra, en especial el menos calificado. Esta situación explica en parte el por qué de la baja productividad de la PYMI, dado que su área de operación por excelencia lo constituyen estas industrias.

Otro de los criterios utilizados para medir la competitividad de la PYMl en la región lo constituye el índice que mide la eficiencia en la utilización de los recursos productivos, el cual se expresa a través de la relación existente entre valor agregado y valor bruto de la producción (VAVBP). Según este indicador, en la medida que aumenta esta relación se obtiene una mejora en el uso de los recursos productivos, pues en ella va implicita una mayor creación de valor agregado que surge de una disminución en el peso rela- tivo del consumo intermedio (consumo de materias primas y otros costos de operación) por parte de las empresas (Valecillos, 1994, 80).

Los datos de la figura № 5 muestran durante el período de estudio una caida progresiva en el rendimiento productivo de la industria fabril zuliana, la cual se acentuó en los últimos años. En efecto, la relación VAVVBP disminuyó para el conjunto del sector de 38,12 a 13,76 , observándose que en dicho cambio incidió con mayor fuerza los establecimientos de gran tamaño ya que este indicador pasa de 39,83 a 13,06 . La PYMI también refleja una baja en esta relación; sin embargo, se nota una tendencia menos desfavorable en el uso de los recursos productivos.

El último indicador que muestra la eficiencia en el sector industrial es la relación existente entre el valor de las inversiones y el personal ocupado; midiendo el grado de incorporación de los recursos tecnológicos en las industrias. En la medida que sea más alto significa que las or- 
Figura $\mathrm{N}^{\circ} 5$

Eficiencia en la Utilización de los

Recursos Productivos ${ }^{1)}$

Zulia 1989 - 1994.

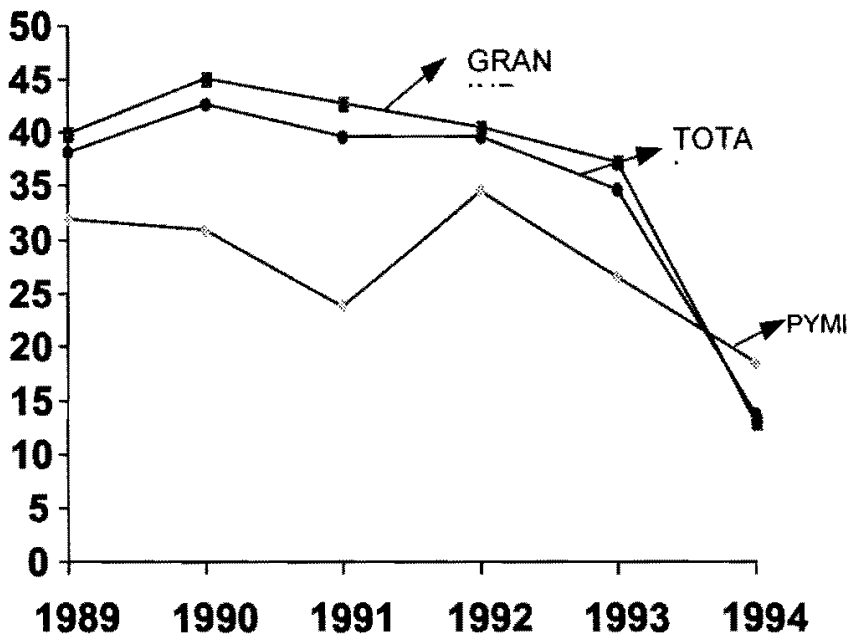

Fuente: Calculos propios en base a la información de la encuesta industrial. Años 1989 - 1994.

1) Relación valor agregado/ valor bruto de la producción.

ganizaciones están incorporando elevados niveles tecnológicos, acordes con sus requerimientos productivos, y por ende, éstas presentan mayor eficiencia y competitividad; mientras que un bajo valor indica que estas empresas son más intensivas en mano de obra, lo que implica que no están adquiriendo los adelantos tecnológicos para realizar las actividades productivas, expresando una estructura fabril atrasada y poco eficiente.

En este sentido, la tabla № 3 pone de manifiesto que para el año 1994 existe una caída pronunciada en este indicador tanto para los grandes establecimientos como para las pequeñas y medianas industrias zulianas. Esta situación revela un deterioro significativo en los parques industriales regionales, $y$ especialmente de la PYMI que no está incorporando las tecnologías necesarias para competir exitosamente en el mercado internacional.

Esta debilidad en el aspecto tecnológico constituye en la actualidad uno de los retos a superar por la industria venezolana y zuliana, pues la dinámica mundial exige cambios profundos en el aparato productivo orientados a la asimilación de tecnologías modernas, dado que el nuevo paradigma económico se fundamenta en la creación de ventajas competitivas creadas por sus pobladores, $y$ en una disminución gradual de las ventajas 
Tabla $N^{2} 3$

Grado de Incorporacion de Recursos Tecnologicos ${ }^{23}$

Zulia $1989-1994$

\begin{tabular}{cccc}
\hline Años & Total & Gran Ind. & PYMI \\
\hline 1989 & 87.43 & 119.11 & 41.38 \\
1990 & 117.34 & 163.77 & 42.98 \\
1991 & 117.94 & 160.70 & 44.85 \\
1992 & 143.45 & 201.91 & 40.85 \\
$1993^{\circ}$ & - & - & - \\
1994 & 74.02 & 09.11 & 21.39 \\
\hline
\end{tabular}

Fuente: Calculos propios en base a la información de la encuesta industrial. Años 1989 - 1994.

1. Sin información.

2. Relación entre inversión y personal ocupado.

comparativas basadas en los recursos naturales.

El estado Zulia posee innumerables ventajas comparativas derivadas de la inmensa cantidad de recursos naturales disponibles, pero su provecho sólo será posible en la medida que sus empresas incorporen los más recientes avances tecnológicos.

Sin embargo, hay que dejar claro que este proceso de modernización industrial no debe limitarse sólo a la asimilación y difusión de las nuevas tecnologias, debe tenerse en cuenta basicamente la incorporación de dos elementos como son la necesidad de crear una capacidad tecnológica propia que permita la participación en el mercado internacional con productos de elevado valor agregado, así como también la necesidad de crear condiciones que permitan la inserción exitosa de la mano de obra local en tales procesos, para lo cual es indispensable el establecimiento de programas de formación y capacitación de los recursos humanos.

\section{Conclusiones}

El estudio elaborado permite constatar que a lo largo del período analizado se experimentó un débil desarrollo de la PYMI zuliana. Los resultados demues. tran que a pesar de que el sector representa la mayor parte de los establecimientos industriales de la región, su aporte al producto es escaso, situación que se explica por la elevada concentración del mismo en la industria tradicional; aspecto éste que se acentúa en el lapso estudiado, determinando un retroceso en la estructura productiva de estas empresas.

En términos generales, los indicadores utilizados para medir la competitividad del sector evidencian el estancamiento productivo en el cual se encuentra. En efecto, se pudo conocer que los niveles de productividad presentan un considerable rezago con respecto al sector 
de la gran industria. Cuestión ésta que se encuentra intimamente vinculado con los elevados niveles de obsolecencia de su parque industrial.

La persistencia de esta situación no sólo las condena al facaso en el mercado mundial, sino que les impone una serie de pruebas dificiles de superar en el mercado interno en vista de la profundización del proceso de apertura comercial.

La problemática anteriormente señalada exige la formulación de políticas que orienten al sector hacia el mejoramiento de la productividad y competitividad. Para ello será necesario cambiar el sesgo social que en torno a ella existe, reconociéndole una mayor posibilidad de respuesta frente a un entorno competitivo, tal como ha sido demostrado en otros países, cuyas políticas han logrado el desarrollo exitoso de la PYMI.

Cabe resaltar, que entre las políticas requeridas para lograr el despegue de la PYMI zuliana, se encuentran:

1. La instrumentación de políticas de capacitación profesional y técnica, orientadas fundamentalmente al fortalecimiento de la educación técnica en la región.

2. Intensificar la actividad gremial con el objeto de lograr un mayor intercambio de información y actualización de los conocimientos y prácticas gerenciales. Esta mayor actividad debe estar orientada al establecimiento de alianzas estratégicas con el sector financiero y el sector científico tecnológico.

3. Se requiere la formación y fortalecimiento de alianzas estratégicas en las diversas ramas de actividad económica en las que opera la PYMI, con la intención de romper barreras existentes para acceder a las innovaciones tecnológicas y a la conquista de nuevos mercados.

Por otra parte, es recomendable la aplicación del benchmarking en la política industrial tomando en cuenta los exitosos resultados obtenidos en Japón y Corea.

Finalmente, es conveniente señalar que los industriales zulianos no deben esperar la solución de todos sus problemas por la vía del Estado, es indispensable poner en práctica los mecanismos de concertación entre ellos mismos para la búsqueda de soluciones conjuntas.

\section{Referencias bibliográficas}

Alvarez, Ruben (1994). Reflexiones en torno a la competitividad de la pequeña y mediana empresa. Revista Investigacióny Gerencia. $N^{2}$ 54. Vol. XI, N²3. Caracas.

Baek, Nakki (1995). Desarrollo e incentivos para las pequeñas y medianas empresas coreanas. Revista Comercio Exterior. Vol 43 №6. México.

Bassetty, Plero (1994). El modelo italiano desde el desarrollo local a la internacionalización de la pequeña y mediana empresa. Banco Interamericano de Desarrollo. México.

Cámara de Industriales del Zulia. Lineamientos para una política industrial. Región zuliana. Maracaibo.

Garcia, Humberto (1994) ¿Una política Industrial para Venezuela? Centro de Estudios del desarrollo (Cendes). Caracas.

Henriquez, Lissette. (1993). Chile: experiencia exportadora de las empresas pequeñas y medianas. Revista Comercio Exterior. Vol. 43, № 6 . 
Hosono, Akio (1992). Pequeñas y medianas empresas en el desarrollo económico de Japón. Banco Interamericano de Desarrollo. México.

Morales, Mariher. (1996) Problemática tecnológica y financiera de la pequeña y mediana industria (PYMI) en la región zuliana. Período 1989 - 1995. Universidad del Zulia. Tesis de Grado. $127 \mathrm{pág}$.

Pizani, Leonardo (1994). La hora de la pequeña y mediana industria. Revista del Centro Nacional de Calidad y Productividad de la Pequeña y Mediana Industria (CEPROPYMI). Año 1, $N^{2} 1$. Caracas, octubre - noviembre.

Rosales, Ramón. (1996). Estrategias para la pequeña y mediana empresa. Edj. ciones IESA. Fundación Polar. Caracas.

Romero, Jenny (1994). Propuesta para meJorar el financiamiento de las mi- croempresas del sector informal. Universidad del Zulia. Facultad de Ciencias Económicas y Sociales. Instituto de Investigaciones.

Romero, Jenny y Acosta, Ana (1997). Situación y Perspectiva de la Pequeña y Mediana Industria (PYMI) en Ia Región Zuliana. Periodo 1989 - 1994. Universidad del Zulia. Facultad de Ciencias Económicas y Sociales. Instituto de Investigaciones.

Sánchez, Fernando. (1993). Acciones en favor de las micros, pequeñas y medianas industrias en México. Revista Comercio Exterior. Vol 43, № 6.

Valecillos, Hector. (1994). Reajuste estructural de la economia y desindustrialización. Revista Banco Central de Venezuela. Vol. 8, número especial .

Yokokura, Takashi. (1984). Empresas pequeñas y medianas. Experiencia Japonesa. Editorial Universidad de Tokio. 